PROCEEDINGS OF THE

AMERICAN MATHEMATICAL SOCIETY

Volume 134, Number 1, Pages 93-98

S 0002-9939(05)07946-3

Article electronically published on June 14, 2005

\title{
A STABILITY PROPERTY FOR LINEAR GROUPS
}

\author{
MARIAN F. ANTON
}

(Communicated by Paul Goerss)

\begin{abstract}
For suitable rings of integers $R$, we show that the $\bmod p$ group cohomology for $G L_{n+3 p-5}(R)$ comes from $G L_{\infty}(R)$ when restricted to the diagonal matrices $D_{n}(R)$ for all ranks $n \geq 2$.
\end{abstract}

\section{INTRODUCTION}

For a prime $p$ and a primitive $p$-th root of unity $\zeta_{p}$, the $\bmod p$ group cohomology of invertible $n \times n$ matrices $G L_{n}$ over $R=\mathbb{Z}\left[\frac{1}{p}, \zeta_{p}\right]$ for $n=1,2, \ldots, \infty$ plays a central role in the algebraic $K$-theory of integers being one of the interfaces for Quillen-Lichtenbaum conjectures [5] (compare also [11]).

A somewhat surprising result [2] about this cohomology for $p$ regular is the fact that its image on diagonal matrices $D_{n}$ inside $G L_{n}$ agrees with the one predicted by the above-mentioned conjectures as soon as a distinguished finite set of $\bmod p$ homology classes vanish in $H_{*} G L_{2}$. The notation $H_{*}$ stands for the mod $p$ group homology functor. These classes were called "étale obstruction classes" due to the fact that they measure the difference between the classifying space of $G L_{n}$ and its étale model in a sense made precise by the Theorem 2.4.

In this note we show that if these étale obstruction classes vanish only in the stable range, then the $\bmod p$ group cohomology for $G L_{n+3 p-5}$ can be calculated when restricted to the diagonal matrices $D_{n}$ instead of $D_{n+3 p-5}$. Although this result is known in the stable range [10] and it is weaker than similar results [1, 9] for $p=2$ and $p=3$, it has the advantage that it holds in the unstable range for any regular prime $p \geq 5$, a case previously unknown. Whether the étale obstruction classes are vanishing already in $H_{*} G L_{2}$ or not for $p \geq 5$, it seems to be a difficult computational question which remains unsolved.

In more detail, by using Dirichlet's Unit Theorem and standard group cohomology calculations for $p$ odd, we have

$$
H^{*} D_{n} \approx \mathbb{F}_{p}\left[x_{1}, x_{2}, \ldots, x_{n}\right] \otimes \bigotimes_{i=0}^{\frac{p-1}{2}} \Lambda\left(y_{i, 1}, y_{i, 2}, \ldots, y_{i, n}\right),
$$

where $H^{*}$ denotes the mod $p$ group cohomology functor and $\Lambda$ the exterior algebra over the finite field $\mathbb{F}_{p}$ of order $p$. The generators have the cohomological degrees given by $\left|x_{j}\right|=2$ and $\left|y_{i, j}\right|=1$ for all $j=1,2, \ldots, n$ and $i=0,1,2, \ldots, \frac{p-1}{2}$. Inside

Received by the editors September 3, 2004 and, in revised form, September 15, 2004.

2000 Mathematics Subject Classification. Primary 19D55, 20G30.

(C)2005 American Mathematical Society 
this ring we distinguish a subring of invariants defined by the formula

$$
H^{*} D_{n}^{i n v}=\mathbb{F}_{p}\left[c_{1}, c_{2}, \ldots, c_{n}\right] \otimes \bigotimes_{i=0}^{\frac{p-1}{2}} \Lambda\left(e_{i, 1}, e_{i, 2}, \ldots, e_{i, n}\right)
$$

with $c_{j}$ the symmetrization of $x_{1} \ldots x_{j}$ and $e_{i, j}$ the symmetrization of $x_{1} \ldots x_{j-1} y_{i, j}$ for all $j=1,2, \ldots, n$ and $i=0,1,2, \ldots, \frac{p-1}{2}$. The main result is

Theorem 1.1. If $p$ is an odd regular prime, then the image of the restriction map $H^{*} G L_{n+3 p-5} \rightarrow H^{*} D_{n}$ induced by the tail inclusion $D_{n} \rightarrow G L_{n+3 p-5}$ (see (2.3)) agrees with $H^{*} D_{n}^{\text {inv }}$ for all $n \geq 2$.

The idea for proving Theorem 1.1 is to use the case $n=\infty$ to show that the étale obstruction classes vanish in the stable range and to slightly modify our theorem, stating that the image of the restriction map is computable if these classes vanish. The étale obstruction classes will be defined and studied in section 2 and the proof of the theorem will be given in section 3 .

Notation 1.2. For the rest of the paper we assume that $p$ is an odd regular prime if not otherwise stated. Let $H^{*}$ (resp. $H_{*}$ ) be the $\bmod p$ cohomology (resp. homology) functor on groups or spaces and let $S L_{n}$ be the subgroup of matrices of determinant 1 in $G L_{n}$ for $n=1,2, \ldots, \infty$.

\section{2. ÉtALE OBStruCtion CLASSES}

2.1. Definition. By setting $n=1, r=\frac{p-1}{2}, x=x_{1}$ and $y_{i}=y_{i, 1}$ in formula (1.1), we see that an additive basis for $H^{*} D_{1}$ is given by the monomials

$$
x^{I}=x^{k} \prod_{i=0}^{r} y_{i}^{\epsilon_{i}}, \quad I=\left(k, \epsilon_{0}, \epsilon_{1}, \ldots, \epsilon_{r}\right),
$$

where $I$ runs over all the sequences of non-negative integers with $\epsilon_{i} \in\{0,1\}$ for $i=0,1,2, \ldots, r$. Let $\left(u_{I}\right)$ be the basis of $H_{*} D_{1}$ dual to the basis $\left(x^{I}\right)$ in $H^{*} D_{1}$. In what follows we tacitly assume that $x$ is a Bockstein class.

Definition 2.1. We call $u_{I} \in H_{*} D_{1}$ with $I$ as in (2.1) an étale obstruction class if

$$
a(I)=\frac{1}{2}\left(\epsilon_{0}+\epsilon_{1}+\ldots+\epsilon_{r}-k\right)
$$

is a positive integer.

Remark 2.2. In the original definition [2] we used $S L_{2}$ instead of $D_{1}$, but the two definitions agree with respect to a suitable group homomorphism $D_{1} \rightarrow S L_{2}$.

2.2. The classifying space étale model. For each $n=2,3, \ldots, \infty$ we have the following commutative diagram:

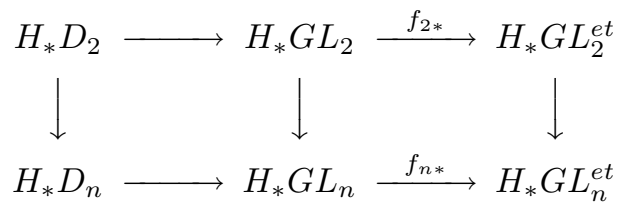

with the first two vertical maps induced by the tail inclusions

$$
D_{2} \rightarrow D_{n}, \quad G L_{2} \rightarrow G L_{n}, \quad A \mapsto\left(\begin{array}{cc}
A & 0 \\
0 & 1
\end{array}\right),
$$


and the unlabelled horizontal maps induced by the canonical inclusions

$$
D_{2} \subset G L_{2}, \quad D_{n} \subset G L_{n} .
$$

The maps $f_{2 *}$ and $f_{n *}$ are induced by the natural Dwyer-Friedlander maps

$$
f_{2}: B G L_{2} \rightarrow B G L_{2}^{e t}, \quad f_{n}: B G L_{n} \rightarrow B G L_{n}^{e t}
$$

from the classifying spaces $B G L_{2}$ and $B G L_{n}$ to their "étale model spaces" $B G L_{2}^{e t}$ and $B G L_{n}^{e t}$, respectively. The notation $H_{*} G L_{n}^{e t}$ stands for $H_{*} B G L_{n}^{e t}$. Their definition can be found elsewhere [4]. Finally, the last vertical map is induced by naturality. In this setting, the following result is known:

Theorem $2.3([6])$. The map $H^{*} G L_{n}^{e t} \rightarrow H^{*} D_{n}$ dual to the composition map

$$
H_{*} D_{n} \rightarrow H_{*} G L_{n} \stackrel{f_{n *}}{\longrightarrow} H_{*} G L_{n}^{e t}
$$

in diagram (2.2) is injective, and its image agrees with $H^{*} D_{n}^{i n v}$ as in (1.2) for all $n=1,2, \ldots, \infty$.

\subsection{The kernel of the composition map $H_{*} D_{n} \rightarrow H_{*} G L_{n}^{e t}$. Let}

$$
t: D_{1} \rightarrow D_{2}, \quad t(a)=\left(\begin{array}{cc}
a^{-1} & 0 \\
0 & a
\end{array}\right), \text { for } a \in D_{1},
$$

be a group homomorphism and consider the diagram

$$
D_{1} \times D_{1} \stackrel{t \times I d}{\longrightarrow} D_{2} \times D_{1} \stackrel{\mu}{\longrightarrow} D_{2}
$$

where $\mu$ is the multiplication of a matrix by a scalar map. Now apply the mod $p$ homology functor $H_{*}$ to this diagram and get

$$
H_{*} D_{1} \otimes H_{*} D_{1} \stackrel{t_{*} \otimes I d}{\longrightarrow} H_{*} D_{2} \otimes H_{*} D_{1} \stackrel{\mu_{*}}{\longrightarrow} H_{*} D_{2} .
$$

$H_{*} D_{1}$ is a Pontryagin ring [3] with the identity element $1 \in H_{0} D_{1} \approx \mathbb{F}_{p}$ corresponding to the identity element of the coefficient field. Then the last map in the above diagram gives $H_{*} D_{2}$ a module structure over the Pontryagin ring $H_{*} D_{1}$ :

$$
w \circ z=\mu_{*}(w \otimes z) \text { for } w \in H_{*} D_{2} \text { and } z \in H_{*} D_{1} \text {. }
$$

Now recall that $u_{I} \in H_{*} D_{1}$ is the dual basis of the additive basis $x^{I} \in H^{*} D_{1}$ as in (2.1). Let $z_{1} \in H_{*} D_{1}$ be an arbitrary element and observe that the image of the element $u_{I} \otimes z_{1} \in H_{*} D_{1} \otimes H_{*} D_{1}$ via the composite map of the diagram (2.4) is $t_{*}\left(u_{I}\right) \circ z_{1} \in H_{*} D_{2}$.

Theorem 2.4. In the unstable range $2 \leq n<\infty$, the kernel of the composition map $H_{*} D_{n} \rightarrow H_{*} G L_{n}^{e t}$ in the diagram (2.2) is spanned by elements of the form

$$
\left(t_{*}\left(u_{I}\right) \circ z_{1}\right) \otimes z_{2} \otimes \ldots \otimes z_{n-1} \in H_{*} D_{2} \otimes H_{*} D_{1} \otimes \ldots \otimes H_{*} D_{1} \approx H_{*} D_{n},
$$

where $u_{I} \in H_{*} D_{1}$ runs over all étale obstruction classes and $z_{i} \in H_{*} D_{1}$ are arbitrary elements for $i=1,2, \ldots, n$.

The proof of this theorem is given in my previous paper [2]. If we specialize to $n=2$ and $z_{1}=1$, then we deduce as a corollary of this theorem that

Corollary 2.5. If $u_{I} \in H_{*} D_{1}$ is an étale obstruction class, then $t_{*}\left(u_{I}\right) \in H_{*} D_{2}$ maps to zero in $H_{*} G L_{n}^{e t}$ via composite maps in the diagram (2.2) for $n=2,3, \ldots, \infty$.

In the stable range, we have Mitchell's result:

Theorem 2.6 ([10]). The kernel of the map $H_{*} D_{\infty} \rightarrow H_{*} G L_{\infty}^{\text {et }}$ is the same as the kernel of the map $H_{*} D_{\infty} \rightarrow H_{*} G L_{\infty}$. 


\section{Proof of the MAIN TheOREM}

3.1. Proof of Theorem 1.1. In the following commutative diagram, in which the maps are defined as in (2.2), we observe that the map $H_{*} G L_{n}^{e t} \rightarrow H_{*} G L_{n+3 p-5}^{e t}$ is injective by Theorem 2.3 and formula (1.2):

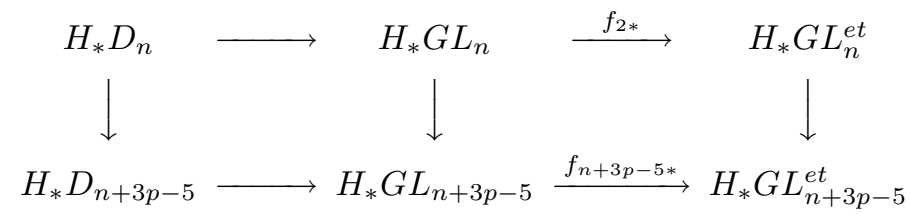

Hence, Theorem 1.1 follows from Theorem 2.3 and the dual of the following statement: the kernel of the composition map $H_{*} D_{n} \rightarrow H_{*} G L_{n}^{e t}$ is included in the kernel of the map $H_{*} D_{n} \rightarrow H_{*} G L_{n+3 p-5}$ for $n \geq 2$. The statement is an immediate consequence of Theorem 2.4 and Lemma 3.4 below.

Let us name the tail inclusions (2.3) simply by "tail".

Lemma 3.1. If $n \geq 3 p-4$, then the element tail $_{*}\left(t_{*}\left(u_{I}\right)\right) \in H_{*} D_{n}$ maps to zero in $H_{*} G L_{n}$ via the map induced by inclusion if $u_{I}$ is an étale obstruction class.

Proof. The key observation is the fact that the homological degree of an element $u_{I}$ with $a(I)$ a positive integer is bounded from above by $\frac{3}{2}(p-1)-1$ (coincidentally, this number is exactly one less the virtual cohomological dimension of $\left.S L_{2}\right)$. This is easily seen by inspection, where $a(I)$ is given in Definition 2.1. By Van der Kallen stability theorem [8], $H_{i} G L_{\infty} \approx H_{i} G L_{n}$ for $n \geq 2 i+1$. Because $\operatorname{tail}_{*}\left(t_{*}\left(u_{I}\right)\right) \in H_{*} D_{n}$ maps to zero in $H_{*} G L_{\infty}^{e t}$ by Corollary 2.5, then Theorem 2.6 now guarantees that tail ${ }_{*}\left(t_{*}\left(u_{I}\right)\right)$ maps to zero in $H_{*} G L_{\infty}$ via maps induced by tail and canonical inclusions. Combining this fact with the previous observation the conclusion follows.

For each $k \geq 2$, the commutative diagram

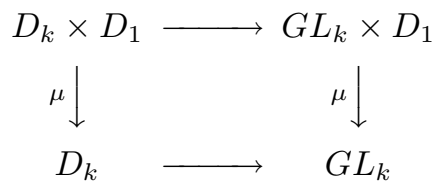

where $\mu$ is the matrix by scalar multiplication map and the horizontal maps are inclusions, induces a homomorphism $H_{*} D_{k} \rightarrow H_{*} G L_{k}$ of modules over the Pontryagin ring $H_{*} D_{1}$. Also, we recall that the Pontryagin ring $H_{*} D_{1}$ for abelian groups like $D_{1}$ is the tensor product of an exterior algebra in degree one generators and a divided polynomial algebra in even degree generators [3], p. 126.

Lemma 3.2. If $w \in H_{*} D_{2}, z, z_{1}, \ldots, z_{k-1} \in H_{*} D_{1}$ with $z$ primitive, and $d^{k}: D_{1} \rightarrow$ $D_{k}$ is the diagonal map, then we have the following formula:

$$
\begin{array}{r}
\left(\left(w \circ z_{1}\right) \otimes z_{2} \otimes \ldots \otimes z_{k-1}\right) \circ z=\left(\left(w \circ z_{1}\right) \otimes z_{2} \otimes \ldots \otimes z_{k-1}\right) \cdot d_{*}^{k}(z) \\
=\left(w \circ\left(z_{1} \cdot z\right)\right) \otimes z_{2} \otimes \ldots \otimes \\
z_{k-1}+\left(w \circ z_{1}\right) \otimes\left(z_{2} \cdot z\right) \otimes \ldots \otimes z_{k-1} \\
+\ldots+\left(w \circ z_{1}\right) \otimes z_{2} \otimes \ldots \otimes\left(z_{k-1} \cdot z\right) .
\end{array}
$$

Here $\circ$ is the module structure product and · is the Pontryagin product in $H_{*} D_{1}$ or $H_{*} D_{k}$ from the context (compare (2.5D). The factor $z$ in this summation is permuted cyclically due to the way the diagonal acts on primitives [7], pp. 284-286. The proof is evident and hence, omitted. 
Lemma 3.3. If $n \geq 3 p-3$, then tail $_{*}\left(t_{*}\left(u_{I}\right) \circ z_{1}\right) \in H_{*} D_{n}$ vanishes in $H_{*} G L_{n}$, where $u_{I} \in H_{*} D_{1}$ is an étale obstruction class and $z_{1} \in H_{*} D_{1}$ is an arbitrary element.

Proof. If we specialize the formula in Lemma 3.2 to $k=n \geq 3 p-3, w=t_{*}\left(u_{I}\right)$, and $z_{i}=1$ for $i=1, \ldots, k-1$, then we see that the left-hand side vanishes in $H_{*} G L_{n}$ by Lemma 3.1 and the observation before Lemma 3.2, while the right-hand side can be written as

$$
\operatorname{tail}_{*}\left(t_{*}\left(u_{I}\right) \circ z\right)+t_{*}\left(u_{I}\right) \otimes d_{*}^{n-3}(z) \otimes 1+\operatorname{tail}_{*}\left(t_{*}\left(u_{I}\right)\right) \otimes z .
$$

By applying Lemma 3.1 and using a block-multiplication argument, we see that $\operatorname{tail}_{*}\left(t_{*}\left(u_{I}\right)\right) \otimes z$ vanishes in $H_{*} G L_{n}$ for $n-1 \geq 3 p-4$. Also, $t_{*}\left(u_{I}\right) \otimes d_{*}^{n-3}(z) \otimes 1$ vanishes in $H_{*} G L_{n}$ because it is a sum of terms invariant under permutations (maps induced from conjugation in $\left.G L_{n}\right)$ and hence, each such summand has the same image as tail ${ }_{*}\left(t_{*}\left(u_{I}\right)\right) \otimes z$ in $H_{*} G L_{n}$, i.e. zero. We conclude that tail $l_{*}\left(t_{*}\left(u_{I}\right) \circ z\right)$ vanishes in $H_{*} G L_{n}$ for $z$ primitive in $H_{*} D_{1}$, and the result follows by an inductive argument.

Lemma 3.4. If $n \geq 2$, then all the elements of the form

$$
\left(t_{*}\left(u_{I}\right) \circ z_{1}\right) \otimes z_{2} \otimes \ldots \otimes z_{n-1} \in H_{*} D_{2} \otimes H_{*} D_{1} \otimes \ldots \otimes H_{*} D_{1} \approx H_{*} D_{n}
$$

vanish in $H_{*} G L_{n+3 p-5}$, where $u_{I} \in H_{*} D_{1}$ is an étale obstruction class and $z_{i} \in$ $H_{*} D_{1}$ are arbitrary elements for $i=1,2, \ldots, n-1$.

Proof. The images in $H_{*} G L_{n+3 p-5}$ of the elements as in the lemma are the same as the images of the elements

$$
z_{2} \otimes \ldots \otimes z_{n-1} \otimes \operatorname{tail}_{*}\left(t_{*}\left(u_{I}\right) \circ z_{1}\right) \in H_{*} D_{n-2} \otimes H_{*} D_{3 p-3},
$$

where tail ${ }_{*}\left(t_{*}\left(u_{I}\right) \circ z_{1}\right) \in H_{*} D_{3 p-3}$ vanishes in $H_{*} G L_{3 p-3}$ by Lemma 3.3 . The proof is now concluded by a block-multiplication argument.

\section{REFERENCES}

1. Marian F. Anton, On a conjecture of Quillen at the prime 3, J. Pure Appl. Algebra 144 (1999), 1-20. MR2000m:19003

2. _ An elementary invariant problem and general linear group cohomology restricted to the diagonal subgroup, Trans. Amer. Math. Soc. 355 (2003), 2327-2340 (electronic). MR 2004c:57061

3. Kenneth S. Brown, Cohomology of groups, Graduate Texts in Mathematics, vol. 87, SpringerVerlag, New York, 1994. MR96a:20072

4. William G. Dwyer, and Eric M. Friedlander, Algebraic and etale K-theory, Trans. Amer. Math. Soc. 292 (1985), 247-280. MR87h:18013

5. W. G. Dwyer and E. M. Friedlander, Conjectural calculations of general linear group homology, Applications of Algebraic $K$-theory to Algebraic Geometry and Number Theory, Part I, II (Boulder, Colo., 1983), Contemp. Math., vol 55, Amer. Math. Soc., Providence, RI, 1986, 135-147. MR88f:18013

6. William G. Dwyer and Eric M. Friedlander, Topological models for arithmetic, Topology 33 (1994), 1-24. MR 95h:19004

7. Allen Hatcher, Algebraic topology, Cambridge University Press, Cambridge, 2002. MR:2002k:55001

8. Wilberd van der Kallen, Homology stability for linear groups, Invent. Math. 60 (1980), 269295. MR 82c:18011

9. Stephen A. Mitchell, On the plus construction for BGL Z $\left[\frac{1}{2}\right]$ at the prime 2, Math. Z. 209 (1992), 205-222. MR93b:55021 
10. Units and general linear group cohomology for a ring of algebraic integers, Math. Z. 228 (1998), 207-220. MR.99k:19002

11. Daniel Quillen, The spectrum of an equivariant cohomology ring. I, II, Ann. of Math. (2) 94 (1971), 549-572; ibid. (2) 94 (1971), 573-602. MR45:7743

Department of Mathematics, University of Kentucky, Lexington, Kentucky 405060027 - AND - I.M.A.R., P.O. Box 1-764, BuCharest, Romania 70700

E-mail address: anton@ms.uky.edu 